In recent development of stereotaxic surgery, the subthalamus has been pai an attention in the anotomico-physiological standpoint. For the treatment 0 involuntary movements: Subthalamotomy (Andy), Campotomy (Spiegel) an Sub-VL-tomy (Narabayashi). Jinnai's Forel-H-tomy, on the other hand, wa aimed for the treatment of epilepsy. In this report, an anatomical review of th Forel $\mathbf{H}$ field was presented in order to clarify our target under such a chaotic stat of surgical denominations, and our clinical experiences of the H-tomy wer presented.

1) A. Forel describes it in Arch. Psychiat. $(7: 393,1877)$ that the $\mathrm{H}$ field it the mediodorsal layer of the Regio subthalamica and located just rostral to the rec nucleus which branches off into $\mathrm{H}^{1}$ and $\mathrm{H}^{2}$ in progress of the rostral direction. The centre of the $\mathrm{H}$ field in the Japanese adult brain is located at $2 \mathrm{~mm}$ caudal anc $4 \mathrm{~mm}$ ventral to the midpoint of the CA-CP line, and $7-8 \mathrm{~mm}$ lateral to the mid. sagittal line, which is a little ventral and caudal to the point given by Schaltenbrand. Bailey.

2) Cryosurgical lesions were made bilaterally in 9 symptomatic and 8 idio. pathic epilepsies, and unilaterally in 2 symptomatic epilepsies. The lesion is $6 \mathrm{~mm}$ in diameter and smaller than the $\mathrm{H}$ field. Seizures were completely abolished ir all of the 2 cases of symptomatic and 3 cases of idiopathic epilepsies in which the $\mathrm{H}$ fields were bilaterally properly destroyed, and one of the 3 cases in which the $\mathrm{H}$ fields were bilaterally properly destroyed, and one of the 3 cases in which the $\mathrm{H}$ fields were properly destroyed in one side and partially in another side. While the target was missed either in one side or both sides, the result was not satisfactory It was stressed that irrespective of idiopathic or symptomatic epilepsies, the lesion should be made at the target in both sides. Seizure discharges become less in frequency and shorter in duration after surgery, but there was no complete disappearance of them in case of complete abolishment of clinical seizure. No complications were found postoperatively at the destruction of the $\mathrm{H}$ field per se.

\title{
36. Surgical Treatment and ACTH-Z Therapy on Infantile Spasm
}

\author{
Yutaka Hori, Shozaburo Utsumi, Shozo Yadoshi, Yoshiaki UedA, \\ Katsuya Shiota, Shigeki Minami, Hideo Sekimoto \\ and Takuko YANASE \\ The 2nd Department of Surgery, Nara Medical College
}

Since several years, we have emphasized that in cerebral palsied patients ther are a number of patients complicated with craniostenotic state and have termed as "sccondary craniostenosis" to differ from congenital craniostenosis. In fact, : 
quarter of the cerebral palsied patients were in the craniostenotic state in which some manifested attacks of infantile spasms. The surgical procedure, craniotomy, were performed on the craniostenotic cerebral palsied patients and resulted in various good effects on motoric and psychic retardation. The most attractive event of the effects was complete disappearance of attacks of infantile spasms.

Of surgically treated craniostenotic patients, 36 infants complained of infantile spasms. Eleven cases of these 36 patients were free from their attacks completely immediately after the surgery. And in other 12 cases their attacks were fairly suppressed but not completely. From these clinical observations, the patients complained of infantile spasms under craniostenotic state are preferable to be treated surgically.

While, the each attack of 12 patients were investigated by means of simultaneous recordings of EEG, EMG and Movies. Based on the investigations, infantile spasm was classified in three types as follows. In type A, the single attack lasted within one second and could be evoked by sound and light stimulation and presented spikes on the electroencephalogram. Type B had attacks lasting 1-10 seconds and the attacks were difficult to be evoked and encephalographically showed no spike. Type $\mathrm{C}$ had long lasting attacks, more than 10 seconds, and was able to be evoked by Bemegride. The attacks of each type responded differently to medication. Type $\mathrm{A}$ and $\mathrm{C}$ responded to anticonvulsants moderately but showed no effects by ACTH-Z. On the other hand, the attacks of type B were controled by ACTH-Z therapy fairly well.

\title{
37. Convulsion evoked by N-Methyl Aspartic Acid
}

Jiro Nagaoka, Nobumoto Saito, Toshio Hirata, Hisao Arai, Hiroshi Sato, Jun Yoneyama and Kei Mryazawa

Dept. of Physiology, Kanagawa Dental College

\section{Clinical and Neurophysiological Investigation of the Preventive Effect to Convulsive Seizure by Means of Administration of GABA ( $\gamma$-aminobutylic acid) Derivatives to CSF Cavity}

\author{
Keizo Matsumoto, Takashi Ohmoto, Shinji Takeuchi, Masaho \\ Murakami, Akira Matsumoto and Masahiro Tanigawa \\ Department of Neurological Surgery, Okayama University Medical School
}

